Revue internationale P.M.E.

Économie et gestion de la petite et moyenne entreprise

\title{
Alexandre Mallard, Petit dans le marché. Une sociologie de la Très Petite Entreprise, Paris, Presses des MINES, 2011, 264 p.
}

\section{Michel Trépanier}

Volume 24, numéro 3-4, 2011

URI : https://id.erudit.org/iderudit/1013672ar

DOI : https://doi.org/10.7202/1013672ar

Aller au sommaire du numéro

Éditeur(s)

Presses de l’Université du Québec

ISSN

0776-5436 (imprimé)

1918-9699 (numérique)

Découvrir la revue

Citer ce compte rendu

Trépanier, M. (2011). Compte rendu de [Alexandre Mallard, Petit dans le marché. Une sociologie de la Très Petite Entreprise, Paris, Presses des MINES, 2011, 264 p.] Revue internationale P.M.E., 24(3-4), 308-311.

https://doi.org/10.7202/1013672ar d'utilisation que vous pouvez consulter en ligne. 


\section{PETIT DANS LE MARCHÉ. UNE SOCIOLOGIE DE LA TRÈS PETITE ENTREPRISE Alexandre Mallard Paris, Presses des MINES, 2011, 264 p.}

Voici un livre fort à-propos puisqu'il est dédié à un type d'entreprise, la très petite, dont l'importance est à la fois «numérique», elles sont très nombreuses et leur nombre va croissant, et «stratégique », puisque dans plusieurs pays industrialisés, chaque crise ou chaque vague de délocalisation conduit de nombreuses personnes à lancer leur propre entreprise afin de se donner les moyens d'exercer leur métier. Mallard porte donc son regard sur la très petite entreprise (TPE); la micro-organisation de dix personnes ou moins, et plus précisément, sur la manière dont celle-ci s'y prend pour construire sa relation au marché dans le contexte de relative rareté des ressources qui est le sien.

Comme on le verra plus loin, l'argumentation développée dans cet excellent livre a une portée qui va au-delà de l'objet étudié dans la mesure où la démarche proposée pourrait être utilisée avec intérêt sur d'autres types d'organisations. Dans ce sens, on aurait aimé que l'auteur confronte sa démarche et ses résultats à la littérature anglo-saxonne qui se déploie le plus souvent sur des paradigmes théoriques et méthodologiques qui, à la lumière des résultats rapportés dans ce livre, paraissent devoir être questionnés. Il est donc malheureux que ces travaux aient été presque complètement passés sous silence.

La manière dont l'auteur circonscrit son objet d'étude mérite d'être soulignée parce qu'elle montre bien en quoi ce travail en apparence anodin joue un rôle central dans la qualité et la pertinence des conclusions auxquelles on pourra arriver au terme d'une étude. D'entrée de jeu, l'auteur indique sa décision de ne pas centrer son analyse sur les TPE de haute technologie qui retiennent tellement l'attention des chercheurs et des organismes publics: ces dernières sont peu représentatives du monde de la TPE et pour comprendre cet univers, mieux vaut s'intéresser à la TPE «ordinaire»; c'est-à-dire aux pratiques les plus usuelles. Mallard indique également que la population désignée par la catégorie «entreprise de dix personnes ou moins» est un agrégat statistique peu pertinent en lui-même puisqu'il regroupe une telle diversité de situations (le petit commerçant, la coiffeuse, le consultant en informatique, le comptable, l'agriculteur, le restaurateur, l'infirmière, l'entrepreneure en biotechnologie, etc.) que la présentation d'un portrait global serait à la fois inutile et erronée. En conséquence, l'analyse qu'il propose respecte l'hétérogénéité des pratiques observées tout en identifiant des sous- 
ensembles qui mettent en évidence des positionnements et font ressortir les axes de différenciation qui structurent cet univers. Finalement, l'auteur fait le pari que l'examen des caractéristiques et des pratiques de la TPE ouvrira la porte à une meilleure compréhension des «modalités contrastées du rapport organisation/marché» (p. 26). C'est un pari qu'il remporte haut la main et qui élargit la portée de son ouvrage.

C'est à l'intérieur de cette perspective que l'auteur aborde les trois thèmes qui constituent les chapitres de son ouvrage: la fabrication des savoirs sur l'environnement économique (chapitre 1), les «capacités commerciales », c'est-à-dire l'assemblage et l'utilisation des ressources dont les TPE ont besoin pour agir sur le marché (chapitres 2 et 3 ), les pratiques de communication et l'usage du téléphone dans les relations au marché (chapitres 4 et 5).

Une autre des qualités de l'ouvrage de Mallard est l'utilisation d'une démarche méthodologique riche et pertinente qui permet non seulement d'atteindre les objectifs visés sur le plan scientifique, mais également de produire des résultats qui ont un potentiel d'utilisation. Soulignons d'abord l'utilisation conjointe de données qualitatives (des entretiens) et quantitatives (observations de trafic téléphonique, sondages téléphoniques). À la différence de beaucoup d'études où les données qualitatives ne servent qu'à préparer les questionnaires qui serviront à la cueillette de données quantitatives qui, elles, seront à la base de l'analyse, on constate ici une réelle intégration des deux types de données. Ainsi, on retrouvera, précisera et validera à plus grande échelle, dans l'analyse quantitative, les types de TPE entrevus dans l'analyse qualitative et cette dernière permettra de mieux comprendre les pratiques qui caractérisent les sous-ensembles identifiés quantitativement. Cette «fusion» des deux types de données et d'analyse permet une compréhension plus fine, plus juste et davantage contextualisée. Ainsi, on a donc des résultats plus intéressants sur le plan scientifique, mais également plus accessibles et plus facilement applicables dans les TPE elles-mêmes. On soulignera ensuite l'excellente utilisation des données qualitatives. Entre autres parce qu'il cherche à identifier des types de TPE, l'auteur prend soin d'indiquer qui parle et d'où, dans cet univers, émane son discours. De la même manière, il indique au lecteur à qui et à quelle proportion de l'échantillon la situation décrite correspond. Somme toute, tant les données utilisées que l'analyse qui en est faite sont riches et détaillées. À ceux qui tendraient à penser que c'est trop, il faut faire remarquer que ce sont là deux qualités qui assurent que l'auteur sait de quoi il parle et qu'elles confèrent une grande crédibilité à son analyse dans la mesure où le lecteur voit clairement et concrètement comment les «choses» fonctionnent et sont structurées. 
Sur le premier thème, «l'expérience marchande», Mallard souligne que dans les TPE, la construction des savoirs sur l'environnement économique se fait dans un rapport de proximité avec les réalités marchandes. Ces dernières sont extrêmement socialisées au sens où il s'agit surtout de contacts directs avec les clients et que la relation sociale qui s'établit entre la TPE et ces derniers joue un rôle récurrent et déterminant dans la compréhension des phénomènes d'attraction et de rétention de la clientèle. Dans la TPE, observe Mallard, la connaissance des réalités marchandes a donc un ancrage géographique et social souvent marqué par la proximité. Cela dit, toutes les TPE ne sont pas identiques et les caractéristiques de leurs activités de même que leur contexte spécifique (le métier exercé, le fait de travailler avec un bien économique particulier et singularisé ou plutôt avec une «marchandise», le fait de travailler à l'échelle locale ou plutôt à l'échelle régionale, etc.) font en sorte que, par exemple, un sous-ensemble de TPE fait davantage appel aux outils classiques du marketing (études de marché, cartographie de la clientèle, etc.) alors que les autres travaillent pour l'essentiel à partir de l'accumulation des expériences vécues.

L'examen des «capacités commerciales» au moyen d'une méthode statistique combinant une analyse des correspondances multiples (ACM) et une analyse typologique permettent à l'auteur de différencier les TPE entre elles et d'identifier les principes structurants de cet univers. L'ACM permet de discerner trois facteurs de différenciation: premièrement, les TPE qui reçoivent leurs clients chez elles (achalandage fort) se distinguent de celles qui se déplacent chez leurs clients (achalandage faible); deuxièmement, les TPE dont les pratiques de recrutement des clients sont peu formalisées (bouche à oreille) apparaissent très différentes de celles où elles le sont fortement (démarchage, publicité, etc.); troisièmement, les TPE de services diffèrent fortement de celles dont l'activité est centrée sur les produits. Comme le souligne Mallard, les variables classiques que sont la taille et le secteur contribuent peu à la structuration de l'univers de la TPE.

L'analyse permet de dégager sept groupes distincts de TPE: les «commerçants de proximité », les «boutiques de produits-services», les «attracteurs», les «prestataires-réseauteurs», les «distanciés prospecteurs», les «entrepreneurs régionaux» et les «concentrés». Sans entrer dans la description détaillée de chacun de ces groupes, retenons simplement que chaque groupe a sa façon propre de capter ses clients (vitrine, bouche à oreille, publicité, utilisation d'intermédiaires), de les rencontrer (nombre de clients, concentration du volume d'affaires), de coproduire avec chacun d'eux les arrangements particuliers de la relation commerciale (type d'activité, part des produits sur mesure dans l'activité), de gérer la temporalité de la relation (fréquence et durée des transactions, temps de travail hebdomadaire) et 
sa dimension géographique (qui se déplace et où). Pour l'auteur, chacun de ces groupes de TPE constitue «une modalité [spécifique] d'exercice de l'activité» et c'est donc, dans chacun des cas, la configuration spécifique des pratiques mises en œuvre et des circonstances dans lesquelles elles le sont qui constituent l'explication des comportements et, possiblement, de la performance. D'une certaine manière, Mallard attire notre attention sur le caractère contextuel de ce qu'est et de ce que fait une TPE.

L'examen des usages du téléphone montre qu'une pratique n'a pas de sens ou d'effet indépendamment des autres pratiques mises en œuvre dans une TPE et que, dans ce sens, elle est «contextuelle». Mallard constate, en effet, que le «téléphone est utilisé [...] assez spécifiquement dans chaque entreprise » (p.198) et que cette utilisation est marquée par l'ensemble des caractéristiques du type de travail commercial qui est le sien. Comme dans le cas des deux thèmes précédents, la taille et le secteur d'activité sont peu utiles pour comprendre les usages du téléphone ainsi que son rôle dans le travail commercial de la TPE.

Partant des conclusions auxquelles il arrive sur les trois thèmes étudiés, le travail de Mallard donne à penser que l'identification de bonnes pratiques au sein d'un échantillon de TPE a peu de pertinence. «La» TPE n'existe pas. En effet, si c'est une configuration de pratiques et un contexte particulier (un métier, un lieu, un type d'activité, etc.) qui définissent une TPE, il en résulte que les pratiques «intéressantes» dans un contexte particulier et associées à d'autres pratiques ne le seront pas nécessairement prises isolément et dans un autre. D'où le peu d'intérêt à «agréger» les TPE et à identifier des facteurs de succès présentés comme «universels» au sein de cette population. Dit autrement, ce ne sont pas les facteurs qui devraient retenir l'attention des chercheurs, mais plutôt les configurations de pratiques ou, comme ledit Mallard, «les modalités d'exercice de l'activité».

Michel Trépanier

Institut national de la recherche scientifique et INRPME/UQTR 